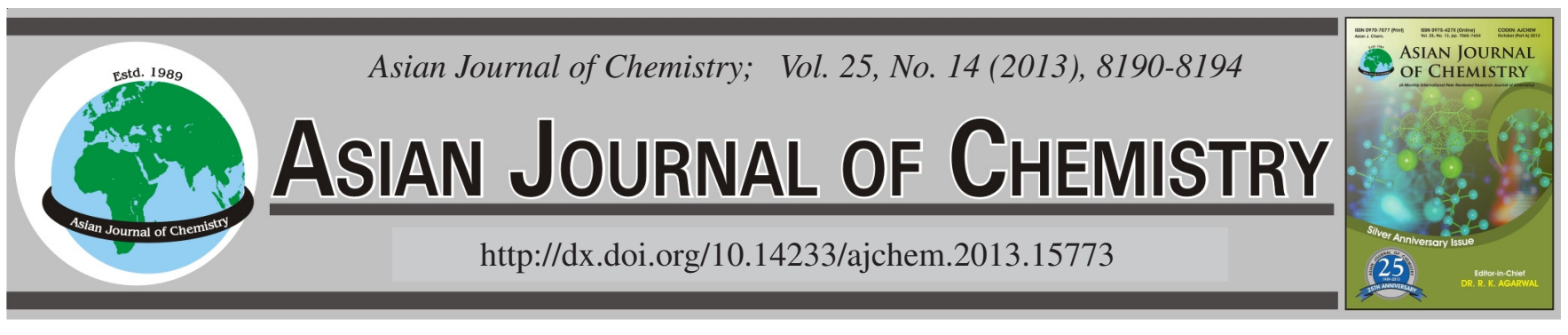

\title{
Quantum Chemistry Calculations on Binding Energies and Orbital Localization of Excitons in Organic Photovoltaic Semiconductors
}

Hui Cao*, Chaozhi Zhang, Tiancheng Feng, Jinguing Chen, Chenbo Fei and Mingxia Song

Key Laboratory of Atmospheric Environment Monitoring and Pollution Control, School of Environment Science and Technology, Nanjing University of Information Science and Technology, Nanjing 210044, Jiangsu Province, P.R. China

*Corresponding author: E-mail: yccaoh@ hotmail.com

(Received: 15 May 2013;

Accepted: 8 August 2013)

AJC-13903

\begin{abstract}
The first principle quantum chemistry calculations is used to investigate the binding energies of excitons and the corresponding orbtial localization of electron and hole in a couple of organic electron donors and electron acceptors. In our calculations, HOMO and LUMO orbitals are exchanged to construct the density matrix and use the quadratically covergent self consistent field method to get the final electronic structures of excitons. Our calculated binding energies of the selected systems are $0.3-0.6 \mathrm{eV}$, in good agreement with the experiments. The orbital localization of electron and hole in excition compared with the ground state is evidently shown with our calculations.
\end{abstract}

Key Words: Exciton, Binding energy, Orbital localization, Organic semiconductor.

\section{INTRODUCTION}

In a typical organic photovoltaic cell ${ }^{1}$, materials of electron donor (D) and electron acceptor (A) are needed for fabricating the D-A heterojunction ${ }^{2-5}$. At the interface, excitons generated by absorption of light dissociate and further separate into free carriers. Excitons can be generated in donor or in acceptor. In the former case, electrons pass through the interface from $\mathrm{D}$ to $\mathrm{A}$, while in the latter case, holes transfer from $\mathrm{A}$ to $\mathrm{D}^{6,7}$. As the first step for understanding the photovoltaic mechanism, detailed information of how the excitons are generated is of important significance ${ }^{8-12}$. In this work, donors and acceptors (Fig. 1) are selected as the prototype to study the electronic properties of excitons.

For the lowest energy photoexcitation, electron in the highest occupied molecular orbital (HOMO) is pumped to the lowest unoccupied molecular orbital (LUMO) and the hole is left in HOMO. Due to the electrostatic attraction between electron and hole, all electronic orbitals relax and the charge density redistributes at the same time. As a result, there is a weak absorption peak before the normal optical absorption, indicating the formation of excitons. The feature of exciton is that electron and hole coulombically attract with each other and thus locate in the energy gap of organic photovoltaic materials. To fully understand the characteristics of excitons, we address two main aspects, namely, the binding energies of excitons and the orbital localization behaviour of excitons in this work. The binding energy for the exciton is much larger than the thermal energy $k_{B} T$ and can greatly influence the exciton dissociation ${ }^{13}$. The degree of spatial localization of orbitals of electron and hole relates to the size of excitons: more localized with smaller size. And the exciton with smaller size means more difficult to be dissociated and separated.

Conventional quantum chemistry calculations on the electronic structure of excitons are based on the combination of multiple single-electron excited configurations. However, this description of electronic structures of excitons is far from the requirement of intuitive understanding. Thus an obvious question arises: is it possible to describe the excitons in a way only one single electronic configuration is involved? In an early theoretical research, Kanai and Grossman ${ }^{4}$ took the approximate density functional theory (DFT) method to calculate the electronic structures of charge transfer excitons in the P3HT/fullerene heterojunction. In this method, a new exchange term is introduced into the approximate density functional to describe the singlet or triplet excitation of molecules $^{15}$. But this method is only implemented with the local density approximation (LDA) ${ }^{16}$ functional. A well known fact is that the local density approximation method often fails to give a satisfactory description of the electronic structures of organic macromolecules. Whereas, the hybrid functional B3LYP ${ }^{16-19}$ overcomes the shortage of local density approximation, since the correct Hartree-Fock exchange potential is introduced. 


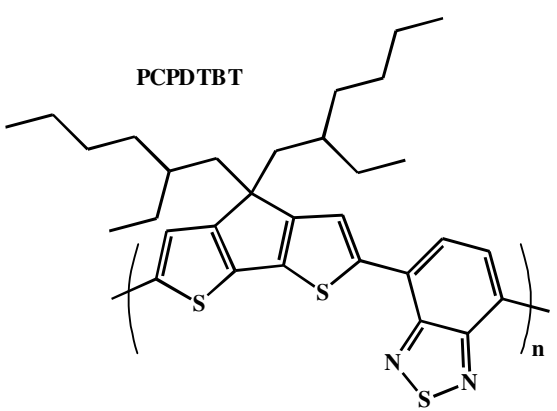<smiles>Cc1ccc(-c2ccc(-c3ccc(-c4cc5c(C)c6sc(C(C)(C)C)cc6c(C)c5s4)s3)c3nn(C)nc23)s1</smiles>
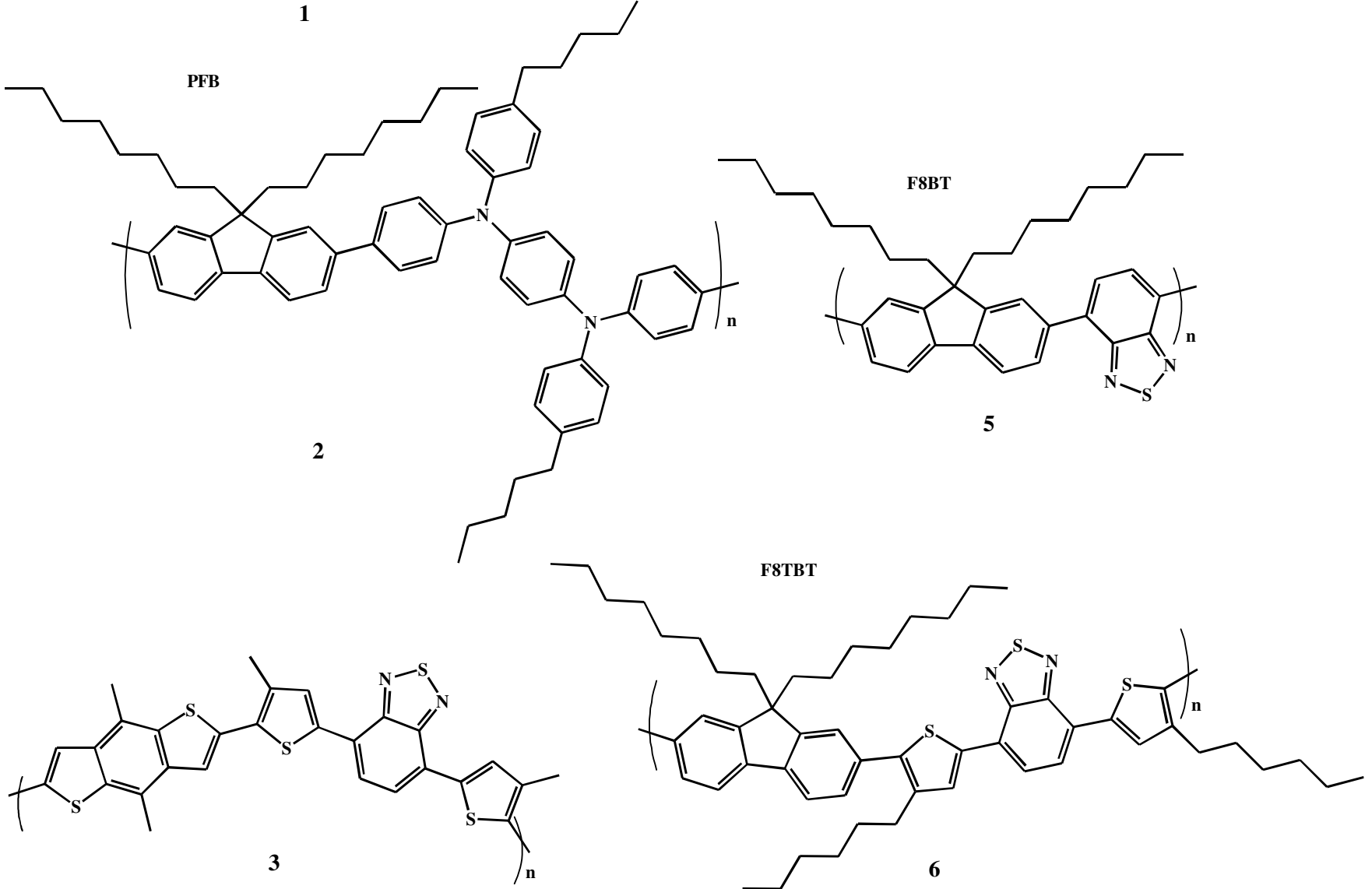

Fig. 1. Structures of the studied electron donors of, $\mathbf{1}$ (PCPDTBT), $\mathbf{2}$ (PFB), $\mathbf{3}, \mathbf{4}$ and the electron acceptors of 5 (F8BT), 6 (F8TBT) oligomers, $\mathrm{n}=2,3,4$ or 5

In this work, we employ the standard DFT method with B3LYP functional to investigate the electronic structures of excitons in organic photovoltaic materials. The key point is to directly excite one electron from occupied orbital to the unoccupied orbital. This is implemented by exchange the corresponding occupied orbital and the unoccupied orbital to construct the new density matrix. After the self consistent process, the electronic energy levels of the original single excited electronic configuration are fully relaxed, giving the correct electronic structures of excitons. Both the singlet and triplet lowest energy excitation are investigated in our paper. In a real organic photovoltaic cell singlet exciton is directly generated by the absorption of incident light and is very important for charge transfer and charge separation ${ }^{20}$, while the triplet exciton is closely related intermediate process of the recombination mechanism ${ }^{11}$. Oligomers with different number of repeating units are taken to investigate the length dependent properties of excitons. We do not carry out the periodic boundary condition (PBC) calculations as Kanai and Grossman ${ }^{14}$ did is due to the fact that in the primitive cell of organic donor of acceptor the orbital is always delocalized. For the purpose to discuss the localization of orbitals of electron and hole in excitons this PBC method is thus not appropriate.

\section{COMPUTATIONAL METHOD}

The geometries of the selected oligomers are optimized by the hybrid density functional B3LYP with the basis set of 6-31G* in the Gaussian 09 package. The electronic properties are calculated for both singlet and triplet excitons with the lowest energy. The calculations of electronic structures for triplet excitons are performed by means of the ordinary triplet setting and an $\alpha$-electron is then excited to the $\beta$-orbital. For singlet exciton calculation, the HOMO and LUMO orbital are exchanged and the quadratically convergent self-consistent- 
field method is employed all the time. The geometrical relaxation $^{21}$ of excitons is neglected due to the consideration that in a superfast charge dissociation and charge separation process at the organic photovoltaic heterojuntion interface this atomic relaxation is not the key process. In other words, in this paper we only take account of the adiabatic process of electronic excitation ${ }^{14}$. In this paper, the binding energies are calculated from the energy difference between the HOMO-LUMO gaps and the excitation energies.

\section{RESULTS AND DISCUSSION}

Binding energies of excitons: Oligomers 1-6 with the repeating units, $\mathrm{n}$, from two to four (or five) are studied and the corresponding binding energies are shown in Table-1. The binding energies of singlet excitons in 1-6 $(n=4$ or 5$)$ are in the range of $0.3-0.6 \mathrm{eV}$, in good agreement with large amount of experimental and theoretical work ${ }^{22,23}$. The energy splitting between the lowest energy singlet and triplet is $c a$. $0.1-0.3 \mathrm{eV}$, indicating that the exchange interaction in 1-6 is much smaller than in PPV, the energy splitting of which is over $0.7 \mathrm{eV}^{24,25}$. In all the six oligomers, the excitonic binding energies of the electronic acceptor, 5 and 6, are bit larger about $0.1 \mathrm{eV}$ than those of the electron donor, 1-4. And the energy splitting between singlet and triplet in 5 and 6 is also larger than that in $1-4$, implying that excitons generated in 5 and 6 are not favourable for the charge dissociation in a real organic photovoltaic heterojunction interface. Therefore, in the photovoltaic heterojunction fabricated by electron donor, 1-4 and electron acceptor, 5 and 6 , the electron (not hole) transfer process is dominate.

\begin{tabular}{|c|c|c|c|c|c|}
\hline \multicolumn{6}{|c|}{$\begin{array}{c}\text { TABLE-1 } \\
\text { BINDING ENERGIES OF EXCITONS GENERATED } \\
\text { IN THE STUDIED ORGANIC PHOTOVOLTAIC } \\
\text { OLIGOMERS (IN THE UNIT OF eV) }\end{array}$} \\
\hline Oligomer & Singlet & Triplet & Oligomer & Singlet & Triplet \\
\hline \multicolumn{3}{|c|}{1 (PCPDTBT) } & \multicolumn{3}{|c|}{4} \\
\hline $\mathrm{n}=2$ & 0.52 & 0.71 & $\mathrm{n}=2$ & 0.52 & 0.71 \\
\hline $\mathrm{n}=3$ & 0.46 & 0.60 & $\mathrm{n}=3$ & 0.46 & 0.62 \\
\hline $\mathrm{n}=4$ & 0.42 & 0.55 & $\mathrm{n}=4$ & 0.42 & 0.57 \\
\hline \multicolumn{3}{|c|}{2 (PFB) } & \multicolumn{3}{|c|}{5 (F8BT) } \\
\hline $\mathrm{n}=2$ & 0.38 & 0.52 & $\mathrm{n}=2$ & 0.54 & 0.85 \\
\hline $\mathrm{n}=3$ & 0.32 & 0.49 & $\mathrm{n}=3$ & 0.47 & 0.55 \\
\hline $\mathrm{n}=4$ & 0.33 & 0.48 & $\mathrm{n}=4$ & 0.42 & 0.67 \\
\hline \multicolumn{3}{|c|}{3} & \multicolumn{3}{|c|}{6 (F8TBT) } \\
\hline $\mathrm{n}=2$ & 0.42 & 0.64 & $\mathrm{n}=2$ & 0.35 & 0.82 \\
\hline $\mathrm{n}=3$ & 0.38 & 0.57 & $\mathrm{n}=3$ & 0.48 & 0.81 \\
\hline $\mathrm{n}=5$ & 0.33 & 0.49 & $\mathrm{n}=4$ & 0.46 & 0.79 \\
\hline
\end{tabular}

Length-dependent behaviour: Table-1 showed that the binding energy becomes smaller with longer oligomer conjugated backbone. This length-dependent behaviour of binding energy is in agreement with one's intuition. When the organic conjugated oligomer is longer, the size of the corresponding exciton is larger. The only one exception is the oligomer 6 with two repeating units, the binding energy of which is bit smaller than those with higher repeating units. This is probably due to the fact that repulsion between the side substituent chains is in part overcome by the sufficient relaxation in space. From the optimized geometry, we find that the distortion of the backbone in oligomer 6 with 2 repeating units is a bit better than with larger repeating units. It is known that the size of the exciton represents the degree of charge diffusion in the oligomer. The charge dissociation process of an exciton begins always from the diffusion of electron and hole in space. Essentially, the binding energy is due to the electrostatic interaction between electron and hole. This electrostatic Coulombical attraction decays rapidly. However, this doesn't mean that in a long oligomer conjugated chain, the exciton will dissociate automatically. In contrast, the exciton becomes stable with a gradually convergent size and binding energy. This is probably due to the existence of the conjugated length in the organic polymer. All the electronic properties, such as the energy levels of HOMO and LUMO and the HOMO-LUMO gap become convergent when the conjugated length is reached.

Electronic properties. The length-dependent binding energy of excitons in organic electron donor and electron acceptor is closely related to the HOMO-LUMO gap and the excitation energies. The HOMO-LUMO gap is corresponding to the optical absorption band while the excitation energy is corresponding to the exciton absorption band in the experiment. When an electron is excited from HOMO to LUMO, a hole is formed in HOMO in order to maintain the electric neutrality. Due to the Coulombical attraction between electron and hole, all the orbials of the system change to a new position. From Table-2, one can find that the energy difference between electron orbital and hole orbital is lower than the HOMO-LUMO energy gap, indicating the Coulombical binding. It is worth to note that from our calculations one can directly get the electron and hole energy level. With the method we employed, in the self-consistent electronic structure an occupied orbital locates at higher position than an unoccupied orbital. For example, the frontier electronic orbitals (both of singlet and triplet) of 6 (F8TBT, $n=4$ ) is shown in Fig. 2. Compared with the ground state electronic structure, all the orbitals in the exciton have relaxed, implying that the exciton is in fact the result of the many-body interaction. The obvious feature of exciton is that electron and hole now reside at the new gap state. The expected behaviour of the gap state is its localization of electrostatic potential distribution.

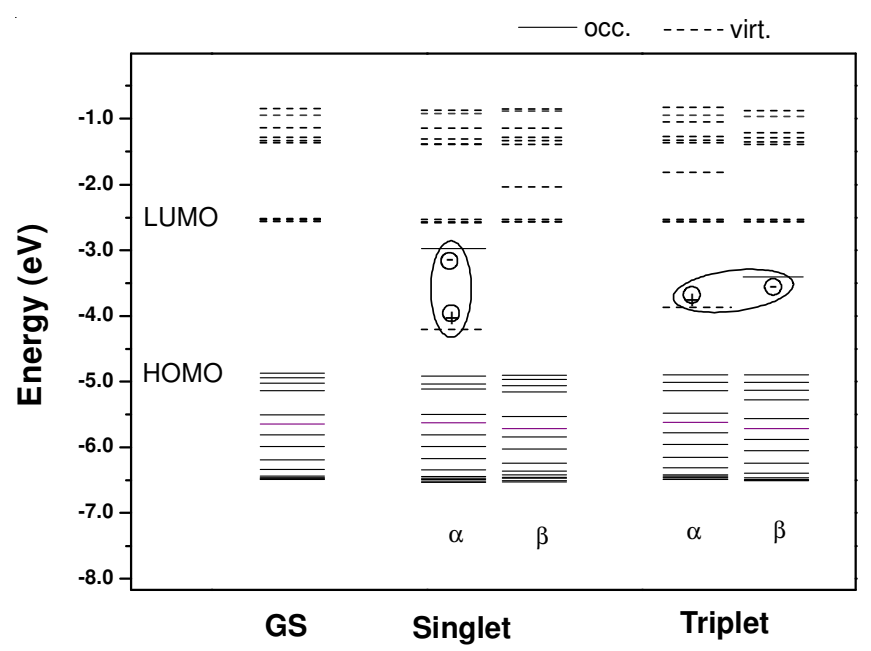

Fig. 2. Frontier electronic orbitals in both singlet and triplet exciton in the representative oligomer $6(\mathrm{~F} 8 \mathrm{TBT}, \mathrm{n}=4)$. The electronic structure of the ground state (GS) system is shown as a comparison 
TABLE-2

EXCITATION ENERGIES $\left(\mathrm{E}_{\mathrm{ex}}\right)$ OF SINGLET STATE $(\mathrm{S})$ AND TRIPLET STATE $(T)$, HOMO-LUMO GAPS $\left(\Delta_{H-L}\right)$ AND ELECTRON-HOLE GAPS $\left(\Delta_{-\mathrm{h}}\right)$ OF SINGLET STATE AND TRIPLET STATE OF THE STUDIED OLIGOMERS (IN THE UNIT OF eV)

\begin{tabular}{|c|c|c|c|c|c|}
\hline Oligomer & $\mathrm{E}_{\mathrm{ex}}(\mathrm{S})$ & $E_{e x}(T)$ & $\Delta_{\mathrm{HL}}$ & $\Delta_{\mathrm{e}-\mathrm{h}}(\mathrm{S})$ & $\Delta_{\mathrm{eh}}(\mathrm{T})$ \\
\hline \multicolumn{6}{|c|}{1 (PCPDTBT) } \\
\hline $\mathrm{n}=2$ & 1.48 & 1.28 & 2.00 & 0.96 & 0.47 \\
\hline $\mathrm{n}=3$ & 1.23 & 1.09 & 1.69 & 0.78 & 0.40 \\
\hline$n=4$ & 1.18 & 1.04 & 1.60 & 0.76 & 0.42 \\
\hline \multicolumn{6}{|c|}{2 (PFB) } \\
\hline $\mathrm{n}=2$ & 2.91 & 2.77 & 3.30 & 2.36 & 1.92 \\
\hline $\mathrm{n}=3$ & 2.94 & 2.76 & 3.26 & 2.45 & 1.91 \\
\hline$n=4$ & 2.91 & 2.76 & 3.24 & 2.37 & 1.91 \\
\hline \multicolumn{6}{|c|}{3} \\
\hline $\mathrm{n}=2$ & 1.61 & 1.40 & 2.04 & 1.10 & 0.52 \\
\hline $\mathrm{n}=3$ & 1.57 & 1.38 & 1.95 & 1.09 & 0.54 \\
\hline $\mathrm{n}=5$ & 1.56 & 1.40 & 1.89 & 1.10 & 0.52 \\
\hline \multicolumn{6}{|c|}{4} \\
\hline $\mathrm{n}=2$ & 1.82 & 1.63 & 2.35 & 1.27 & 0.75 \\
\hline $\mathrm{n}=3$ & 1.77 & 1.60 & 2.23 & 1.25 & 0.77 \\
\hline$n=4$ & 1.76 & 1.60 & 2.18 & 1.26 & 0.77 \\
\hline \multicolumn{6}{|c|}{5 (F8BT) } \\
\hline $\mathrm{n}=2$ & 2.33 & 2.02 & 2.88 & 1.70 & 0.87 \\
\hline $\mathrm{n}=3$ & 2.28 & 2.19 & 2.75 & 1.70 & 1.55 \\
\hline $\mathrm{n}=4$ & 2.29 & 2.04 & 2.72 & 1.75 & 0.90 \\
\hline \multicolumn{6}{|c|}{6 (F8TBT) } \\
\hline $\mathrm{n}=2$ & 2.03 & 1.56 & 2.38 & 1.63 & 0.53 \\
\hline $\mathrm{n}=3$ & 1.82 & 1.50 & 2.31 & 1.22 & 0.47 \\
\hline$n=4$ & 1.83 & 1.51 & 2.30 & 1.23 & 0.47 \\
\hline
\end{tabular}

Electrostatic potential distribution and orbital localization: The electrostatic potential distribution of both the HOMO and LUMO of ground state of $6(n=4)$ are shown in Fig. 3. One can see that the LUMO electronic state distributes over the right three repeating units, while the HOMO electron state distributes over the left three repeating units. After the excitation by the incident light and formation of singlet exciton, the original HOMO and LUMO now become the more localized hole state and electron state (Fig. 4). The electron and hole in the exciton now reside only at the second repeating unit from the right end of oligomer 6. In contrast to the discussion of energy level in the energy space, the description of electrostatic potential distribution is in the 3-dimentional coordinate space. The above mentioned size of exciton is thus clearly shown in Fig. 4. With a close looking at Fig. 4, one can recognize that the electron is now residing at the $\pi$ antibonding orbital while the hole is locating at the $\pi$ bonding orbital.

\section{Conclusion}

In this paper, quantum chemistry calculations are performed to investigate the binding energies of excitons and the corresponding orbtial localization of electron and hole in a series of organic electron donors and electron acceptors, which are taken to fabricate the photovoltaic cells. HOMO and LUMO orbital are exchanged to construct the density matrix and use the quadratically covergent self consistent field method to get the final electronic structures of excitons. This method is proved very stable in getting the excitonic properties. Our calculated binding energies in the range of $0.3-0.6 \mathrm{eV}$ in the
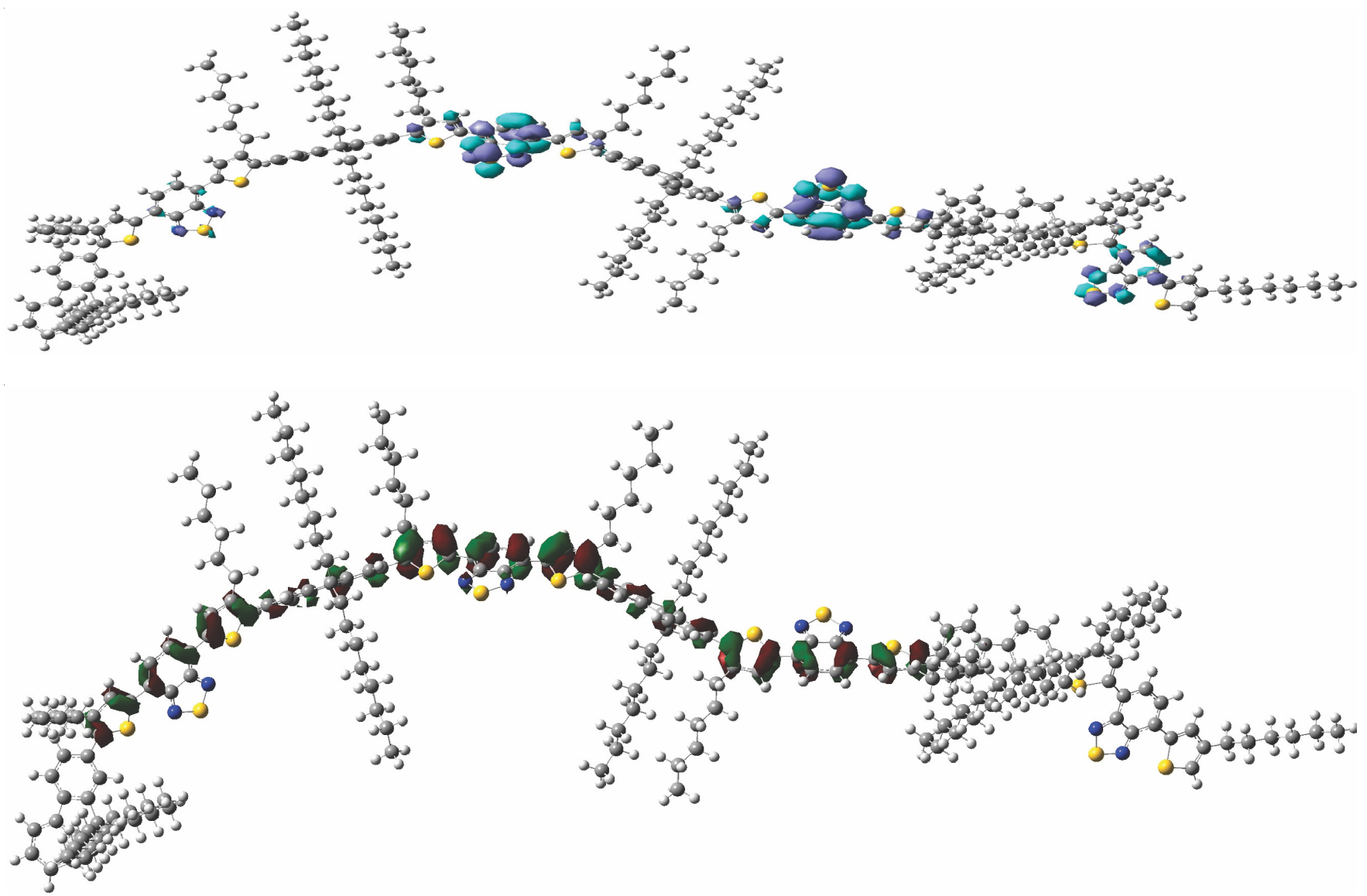

Fig. 3. Electrostatic potential distribution of HOMO and LUMO in the ground state of 6 (F8TBT) 

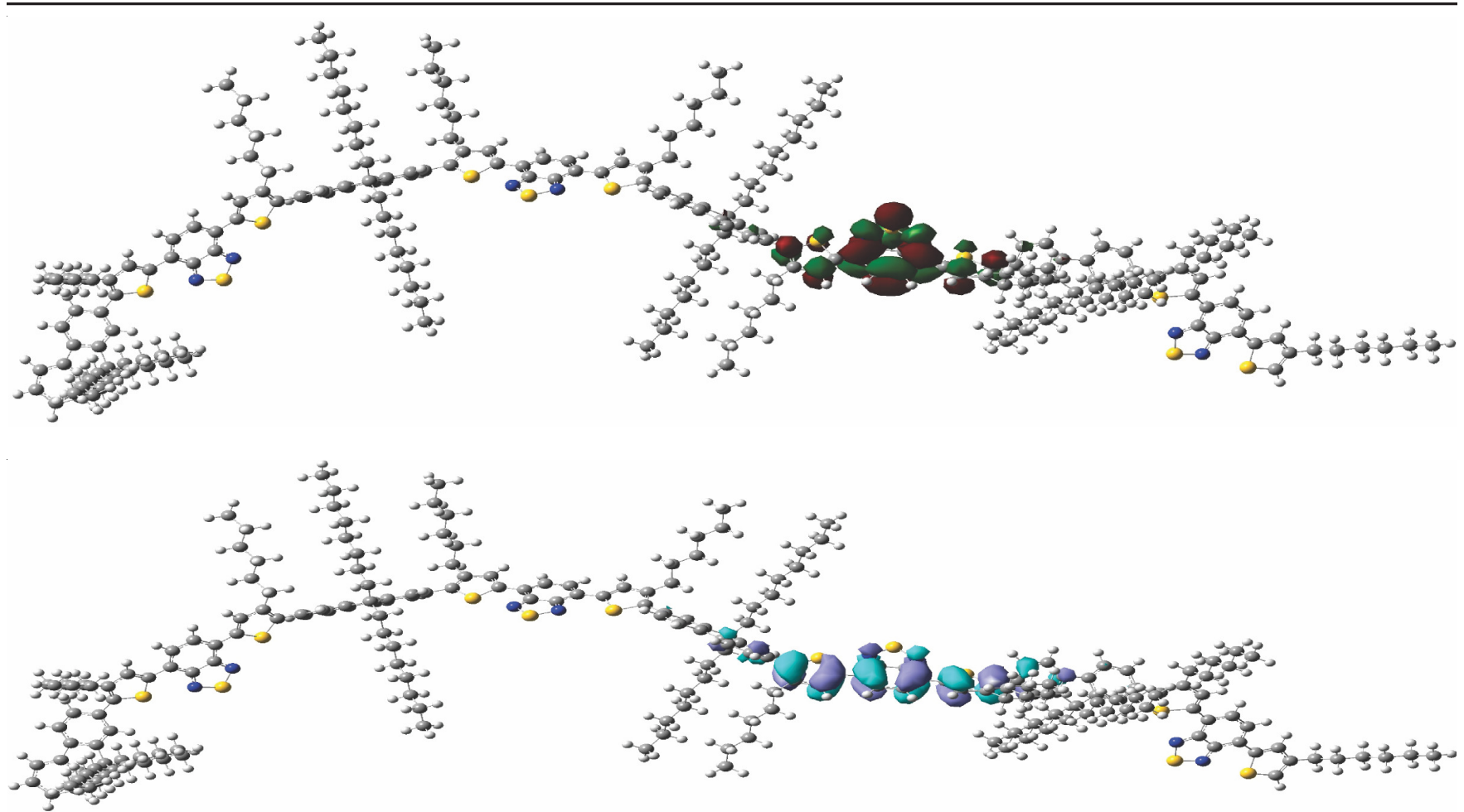

Fig. 4. Electrostatic potential distribution of electron and hole in the singlet exciton of 6 (F8TBT)

selected systems, 1-6, are in good agreement with the experiments and other theoretical calculations. The orbital localization of electron and hole in exciton compared with the ground state is evidently shown with our calculations.

\section{ACKNOWLEDGEMENTS}

The authors gratefully acknowledged the financial support by the NUIST Talents of Introduction Start Fund (S8112005001), the Jiangsu Specially-Appointed Professor Program (R2012T01) and the Foundation for Special Project on the Integration of Industry, Education and Research of Jiangsu Province (BY2012028) and Jiangsu Province Innovation Platform for Superiority Subject of Environmental Science and Engineering.

\section{REFERENCES}

1. N. Agrait, A.L. Yeyati and J.M. ven Ruitenbeek, Phys. Rep., 81, 377 (2003).

2. C.J. Brabec, N.S. Sariciftci and J.C. Hummelen, Adv. Funct. Mater., 11, 15 (2001).

3. K.M. Coakley and M.D. McGehee, Chem. Mater, 16, 4533 (2004).

4. H. Hoppe and N.S. Sariciftci, J. Mater. Res., 19, 1924 (2004).

5. L.Q. Yang, H.X. Zhou, S.C. Price and W. You, J. Am. Chem. Soc., 134, 5432 (2012).
6. J.J.M. Halls, K. Pichler, R.H. Friend, S.C.Moratti and A.B. Holmes, Appl. Phys. Lett., 68, 3120 (1996).

7. M.M. Wienk, J.M. Kroon, W.J.H. Verhees, J. Knol, J.C. Hummelen, P.A.V. Hal and R.A.J. Janssen, Angew. Chem. Int. Ed., 42, 3371 (2003).

8. M.L. Cohen, Ann. Rev. Mater. Sci., 30, 1 (2000).

9. M.L. Cohen, Mater. Sci. Eng. C, 15, 1 (2001).

10. S. Tretiak and S. Mukamel, Chem. Rev. 102, 3171 (2002).

11. J.L Brédas, D. Beljonne, V. Coropceanu and J. Cornil, Chem. Rev, 104, 4971 (2004).

12. G.D. Scholes and G. Rumbles, Nature Mater., 5, 683 (2006).

13. T.M. Clarke and J.R. Durrant, Chem. Rev., 110, 6736 (2010).

14. Y. Kanai and J.C. Grossman, Nano Lett., 7, 1967 (2007).

15. T. Ziegler, Chem. Rev., 91, 651 (1991).

16. S.H. Vosko, L. Wilk and M. Nusair, Can. J. Phys., 58, 1200 (1980).

17. A.D. Becke, J. Chem. Phys., 98, 5648 (1993).

18. A.D. Becke and M.R. Roussel, Phys. Rev. A, 39, 3761 (1989).

19. C. Lee, W. Yang and R.G. Parr, Phys. Rev. B, 37, 785 (1988).

20. A.A. Bakulin, A. Rao, V.G. Pavelyev, P.H.M. van Loosdrecht, M.S. Pshenichnikov, D. Niedzialek, J. Cornil, D. Beljonne and R.H. Friend, Science, 335, 1340 (2012).

21. H. Cao, Q. Zhang and C.Z. Zhang, Asian J. Chem., 25, 2177 (2013).

22. J.L. Brédas, J. Cornil and A.J. Heeger, Adv. Mater, 8, 447 (1996).

23. Z. Shuai, J.L. Brédas and W.P. Su, Chem. Phys. Lett., 228, 301 (1994).

24. Y.V. Romanovskii, A. Gerhard, B. Schweitzer, U. Scherf, R.I. Personov and H. Bässler, Phys. Rev. Lett., 84, 1027 (2000).

25. D. Beljonne, Z. Shuai, G. Pourtois and J.L. Brédas, J. Phys. Chem. A, 105, 3899 (2001). 